\title{
LicenseScript: A Novel Digital Rights Language and its Semantics*
}

\author{
Cheun Ngen Chong ${ }^{1}$, Ricardo Corin ${ }^{1}$, Sandro Etalle ${ }^{1}$, Pieter Hartel ${ }^{1}$, Willem Jonker ${ }^{2}$, and Yee Wei \\ Law $^{1}$ \\ ${ }^{1}$ University of Twente, The Netherlands $\quad{ }^{2}$ Philips Research, The Netherlands \\ \{chong, corin, etalle, pieter,ywlaw\}@cs.utwente.nl, willem.jonker@philips.com
}

\begin{abstract}
We propose LicenseScript as a new multiset rewriting/logic based language for expressing dynamic conditions of use of digital assets such as music, video or private data. LicenseScript differs from other DRM languages in that it caters for the intentional but legal manipulation of data. We believe this feature is the answer to providing the flexibility needed to support emerging usage paradigms of digital data. We provide the language with a simple semantics based on traces.
\end{abstract}

\section{Introduction}

Most information, such as books, music, video, personal data and sensor readings (we generalize this information as data), is intended for a specific use. This specific use should conform to particular terms and conditions, which are often governed by licenses. To describe a license, a specific language is needed. In fact, the last few years have witnessed a proliferation of Digital Rights Languages (DRL). These are usually based on XML, e.g. XrML [8] (www. xrml . org) and ODRL [9] (www . odr l . net).

It is now widely acknowledged that the above-mentioned XML-based DRLs have some important shortcomings: (1) the syntax is complicated and obscure when the conditions of use become complex, (2) these languages lack a formal semantics $[6,12]$; the meaning of licenses relies heavily on the human interpretation, and (3) the language cannot express many useful copyright laws [11].

Gunter et al [6] overcome some of the drawbacks by introducing an abstract model and language with a corre-

* This work has been supported the Telematica Instituut via the LicenseScript project. sponding formal semantics. Pucella and Weissman [12] follow up Gunter et al's effort with more rigor. They reason about the licenses and the user's actions with respect to the licenses; this is done by means of a temporal deontic logic.

However, no DRL introduced so far is flexible enough to accommodate the sophisticated conditions of use that are needed for real use. Consider, for instance, the scenario of Electronic Music Distribution (EMD), where anyone can compose, edit, and distribute music and videos. There is a clear need for a system that allows copyrighted music to be lawfully clipped, mixed, edited, and later be played in public and sold on the market. Additionally, the licensing language should be able to capture the intention of copyright laws, such as: (1) Fair use (reproduction in copies for purposes of education, critiques, etc.), (2) Exemption of Public Display (public display and performance of copyrighted content), and (4) Ephemeral Recordings (for local transmissions, security or archival preservation) among others. These have become one of the main requirements of DRL yet the scope of the current DRLs is limited to rights expression [11].

Authorized Domains Licenses play an important role in the electronic distribution of music. With the advent of the Internet, music labels are searching for ways of distributing music over the Internet in a way that respects the rights of the owners and the labels. At the same time, partly due to Napster, users have become used to easy access to music on their devices. As a result EMD will only be successful if these systems provide flexible support for licensing of music, which in turn requires flexible and easy to understand licenses will a well defined semantics. In an attempt to cope with the requirement of seamless music access on users devices, the notion of authorized domain has been developed (http : //www.dvb.org, [14]). An authorized domain can 
be seen as the collection of devices that belongs to a user or a household. The idea is that music is delivered to the authorized domain, and that it can be accessed seamlessly on any device in that domain. The music access is governed by licenses that are bound to the domain, rather than to individual devices. In addition to licenses that govern the access within the domain, there are licenses that govern the exchange of music between domains. The latter guarantees that unauthorized music exchange can be prohibited.

Regrettably, state-of-the-art languages cannot cope with this scenario.

Contribution of this paper In this paper, we propose $\mathrm{Li}$ censeScript, a language that is able to express conditions of use of dynamic and evolving data in authorized domains. LicenseScript is based on (1) multiset rewriting, which is able to capture the dynamic evolution of licenses, (2) logic programming, which captures the static terms and conditions on a license, and (3) a judicious choice of the interfacing mechanism between the static and dynamic domains. LicenseScript makes it possible to express a multitude of sophisticated usage patterns precisely and clearly. The formal basis of LicenseScript (multiset rewriting and logic programming) provides for a concise and explicit formal semantics.

The organization of the remainder of the paper is as follows: Section 2 explains the LicenseScript language. Section 3 demonstrates examples for Electronic Music Distribution. Finally, Section 4 gives the conclusions and related work.

\section{Language}

In this section we describe the LicenseScript language. We start by introducing some basic concepts that are needed in the sequel.

\subsection{Preliminaries}

As mentioned earlier, LicenseScript is based on multiset rewriting. By a multiset (also known as a bag) we mean a set with possibly repeated elements; denoted by using brackets. For example, $[a, b, b, c]$ is a multiset.

In our approach, licenses are bound to terms that reside in multisets. For the specification of these licenses, we use logic programming; the reader is thus assumed to be familiar with the terminology and the basic results of the semantics of logic programs $[1,10]$. We also use Prolog notation: we use words that start with uppercase $(X, Y, \ldots)$ to denote variables, and lowercase (music_piece, video_track, expires, ...) to denote constants. We work with queries, that is sequences of atoms. Further, given a syntactic construct $E$ (so for example, a term, an atom or a set of equations) we denote by $\operatorname{Var}(E)$ the set of the variables appearing in $E$. Given a substitution $\sigma=\left\{x_{1} / t_{1}, \ldots, x_{n} / t_{n}\right\}$ we denote by $\operatorname{Dom}(\sigma)$ the set of variables $\left\{x_{1}, \ldots, x_{n}\right\}$. A substitution $\sigma$ is called a matching substitution of terms $t$ and $s$ if $t \sigma=s$, and $\operatorname{Dom}(\sigma)=\operatorname{Var}(t)$. In that case, we say that $t$ matches $s$. If a term matches with another one, then it follows that there exists a unique matching substitution.

We also borrow the concept of SLD-resolution from logic programming:

Definition 1. Given a program $P$, and a query (i.e., a conjunction of atoms) $Q$, we write $P \vdash_{S L D} Q$ (or simply $P \vdash Q$ ) when there is a successful SLD-derivation for query $Q$ in program $P$. A succesful execution of a query may result in a (computed answer) substitution.

$P \vdash Q$ basically means that execution of the query $Q$ in the program $P$ yields success.

\subsection{Licenses}

A license defines the terms and conditions of use for music, videos etc. Therefore, a license contains at least two relevant items of information: (i) a reference to the data that is being licensed, and (ii) the conditions of use on that data.

In our formalism, a license is represented by a term of the form lic(content, $\Delta, B$ ) (as can be seen in Figure 1) where:

- content is a unique identifier representing the data the license refers to.

- $\Delta$ is a set of clauses, i.e., a Prolog program. This program defines when certain operations (like play) are allowed.

- $B$ is a set of bindings, i.e., a set containing elements of the form name $\equiv$ value. For instance $\{$ expires $\equiv$ $10 / 10 / 2003\}$ is a set of bindings with just one element.

Bindings are needed to have a flexible way of storing modifiable data. A license could be regarded as a database in which $\Delta$ is the intensional part, while $B$ is the extensional part.

To interface licenses with the external world, we have to define an interface, i.e., a set of reserved calls that form the "API" of the license. The precise definition of this interface is beyond the scope of this paper. For example, we use canplay $(\cdot)$ to indicate when a license allows a given piece of music to be played: if the query canplay $\left(B, B^{\prime}\right)$ succeeds in the program $\Delta$, this means that the license $\operatorname{lic}(a, \Delta, B)$ allows the piece $a$ to be played. Notice that we passed the 


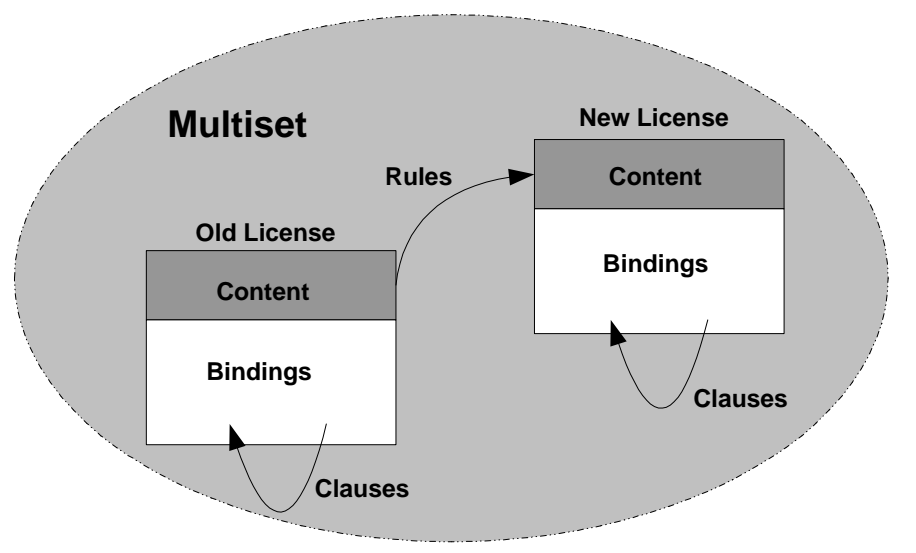

Figure 1. The transformation of licenses with content and bindings in a multiset caused by rules.

set of bindings $B$ as an argument to the query. In the rest of paper we use more reserved calls, which are gathered in Table 1.

\section{Example 2.}

1. The license

$$
\operatorname{lic}(m u s,\{\operatorname{canplay}(X, X):- \text { true. }\},\{\})
$$

allows mus to be played.

2. The license lic(mus, \{\}$,\{\})$ does not allow any operation on mus.

3. The license

$$
\operatorname{lic}(a, \Delta,\{\text { expires } \equiv 10 / 10 / 2003\})
$$

where $\Delta$ is

$$
\begin{aligned}
\{\operatorname{canplay}(B, B):- & \operatorname{today}(D), \\
& \text { get_value }(B, \text { expires, Exp }), \\
& \operatorname{Exp}>D .\}
\end{aligned}
$$

allows to play $a$ until the given expiration day.

$\operatorname{today}(D)$ and get_value $(B, n, V)$ are two primitives that work as follows: $\operatorname{today}(D)$ binds the variable $D$ to the current system date, while get_value $(B, n, V)$ reports in $V$ the value of the name $n$ according to the set of bindings $B$. In the remainder, we gather all such primitives in a special program that we call the domain, denoted $D$. Notice that there can be many domains in which licenses reside, and probably a domain will have different meanings for the primitives than another domain.

\begin{tabular}{|l|l|}
\hline Reserved Call & Description \\
\hline \hline canplay & Decides if the license can be played. \\
validdomain & Checks if the license is valid in the AD. \\
canload & To load some money to the wallet. \\
cantransfer & To transfer money to an entity. \\
canpay & To pay money to an entity. \\
canclip & To clip the license. \\
canrender & Decides if the license can be rendered. \\
\hline
\end{tabular}

Table 1. Reserved calls.

Changing the Bindings There are situations in which the "execution" of a license should be followed by the creation of a new set of bindings for the next step in the evolution of the license. Consider for instance a license that allows to play a piece of music only a given number of times: every time a play action is carried out, a counter should be incremented. This is done by means of the primitive set_value(Oldbindings, name, value, Newbindings).

This primitive allows a name from a binding to be associated with a new value, which we use to support the evolution of licences. Consider, for instance, the following license:

$$
\operatorname{lic}(a, \Delta,\{\text { played_times } \equiv 3\})
$$

where $\Delta$ consists of the following clause:

$$
\begin{aligned}
\operatorname{canplay}\left(B, B^{\prime}\right):- & g e t \_v a l u e(B, \text { played_times }, R), \\
& R<10, \\
& \text { set_value }\left(B, \text { played_times }, R+1, B^{\prime}\right) .
\end{aligned}
$$

Here, we first extract the value of variable played_times into local variable $R$. Then, if we have not exhausted the possible playing times allowed by the license (in this case, 10 ), we proceed to increase the value of playedtimes from bindings $B$ to $R+1$, into new output bindings set $B^{\prime}$. 
In the remainder of the paper, we use more primitives as system calls, as shown in Table 2.

\begin{tabular}{|c|c|}
\hline System Call & Description \\
\hline get_value $(B, n, V)$ & $\begin{array}{l}\text { Report in } V \text { the value of } n \\
\text { from } B \text {. }\end{array}$ \\
\hline set_value $($ OldB, $n, V, N e w B)$ & $\begin{array}{l}\text { Give value } V \text { to } n \text { from } \\
\text { OldB to } N e w B \text {. }\end{array}$ \\
\hline $\operatorname{today}(D)$ & $\begin{array}{l}\text { Bind } D \text { to the current sys- } \\
\text { tem date. }\end{array}$ \\
\hline identify (Id) & $\begin{array}{l}\text { Retrieve the current AD into } \\
I d \text {. }\end{array}$ \\
\hline transfer $(P, M)$ & $\begin{array}{l}\text { Transfer of money } M \text { to en- } \\
\text { tity } P \text {. }\end{array}$ \\
\hline
\end{tabular}

Table 2. System calls.

\subsection{The Rules}

Licenses typically reside inside a device. The modelling of communication between devices and the licenses is done by means of rewrite rules. These rules can be thought as the firmware of the device; licenses may come and go from a device, but the rules are fixed into the device (however, rules can be 'updated' once in a while) .

The syntax of rules we adopt is that of multiset rewriting (we use, in particular, Gamma notation [3, 2]).

Definition 3. A rewrite rule is a 4-tuple

$$
\text { rule }(\text { arg }): \text { lms } \rightarrow \text { rms } \Leftarrow \text { cond }
$$

where rule $(\mathrm{arg})$ is an atom called rule label, $\mathrm{lms}$ and $\mathrm{rms}$ are two multisets, and cond is a sequence of elements of the form $P_{i} \vdash Q_{i}$.

Notice that a substitution $\sigma$ can be applied in a natural way to a rule: $\sigma($ rule $(\arg ): l m s \rightarrow r m s \Leftarrow$ cond $)=$ rule $(\sigma(\arg )): \sigma(l m s) \rightarrow \sigma(r m s) \Leftarrow \sigma($ cond $)$.

Intuitively, rules can be applied to a "target" multiset $M S$, if the following two conditions hold:

- First, the left multiset $l m s$ has to match some sub multiset of $M S$; this sub multiset is to be replaced with (right) multiset rms;

- Second, the conditions cond of the rule must hold; This is done by executing all the queries in cond, and checking that the result is success.

We formalise the meaning of rule execution in the next section. An example for a rule is the following:

$$
\begin{aligned}
\operatorname{play}(X) & : \operatorname{lic}(X, \Delta, B) \rightarrow \operatorname{lic}\left(X, \Delta, B^{\prime}\right) \\
& \Leftarrow \Delta \vdash \operatorname{canplay}\left(B, B^{\prime}\right)
\end{aligned}
$$

This rule can be applied to a license $\operatorname{lic}(X, \Delta, B)$, replacing it with another license $\operatorname{lic}\left(X, \Delta, B^{\prime}\right)$ if condition $\Delta \vdash \operatorname{canplay}\left(B, B^{\prime}\right)$ holds.

\subsection{LicenseScript Execution Model}

As we already mentioned, licenses are represented by terms of the form lic(content, $\Delta, B)$. For the sake of exposure, we assume that all available licenses are stored in a given multiset $M S$.

Definition 4. Rule execution. Given two multisets $M S$ and $M S^{\prime}$, a rule label $: l \rightarrow r \Leftarrow$ cond, and an atom $a$ (called the request action), we write $M S \stackrel{a \sigma}{\longrightarrow} M S^{\prime}$ if:

1. a matches with label, with matching $\sigma_{1}$.

2. $l \sigma_{1}$ matches with $T^{\prime}$, with matching $\sigma_{2}$, for some sub multiset $T^{\prime}$ of $M S$.

3. For each $\Delta_{i} \vdash \phi_{i}, i \in[1, n]$ in cond, $\left(\Delta_{i} \vdash\right.$ $\left.\phi_{i}\right) \sigma_{1} \sigma_{2} \delta_{1} \cdots \delta_{i-1}$ succeeds, with computed answer substitution $\delta_{i}$;

4. $M S^{\prime}$ ' is the result of removing each term in $T^{\prime}$ from $M S$, and appending $r \sigma_{1} \sigma_{2} \delta_{1} \cdots \delta_{n}$ to it.

Step 1 of this definition represents the choice of a rule for executing a given request action $a$ (e.g., play (mus)) from the environment. Notice that there may be more than one rule that matches with the request action, so a first source of non-determinism appears here. The request action can also fail if no rule matches with the request. After a rule is chosen, Step 2 finds a set of licenses $T^{\prime}$ in the multiset MSto whic the rule can be applied. Again, different choices of $T^{\prime}$ may produce non-determinism here. This corresponds to the possible situation in which the user possesses more than one license (or set of licenses) that allows her to effectuate the desired action. In this case, we can assume that the system asks the user which license should be used. Step 3 checks that the conditions of the rule hold, by executing the queries with the carried out substitutions. Finally, Step 4 transforms the multiset by applying the replacement specified by the rule.

Example 5. Consider the multiset containing the following licenses: $M S=[\operatorname{lic}($ music $, \Gamma, C), \operatorname{lic}($ video $, \Sigma, D)]$, where $C=\{$ played_times $\equiv 2\}, D=\{$ played_times $\equiv$ $10\}$, and $\Gamma=\Sigma=$

$$
\begin{aligned}
\left\{\operatorname{canplay}\left(B, B^{\prime}\right):-\right. & \operatorname{get}(B, \text { played_times }, N), \\
& N<10, \\
& \left.\operatorname{set}\left(B, \text { played_times }, N+1, B^{\prime}\right)\right\}
\end{aligned}
$$


Let $R$ be the singleton rule set containing the following rule:

$$
\begin{array}{r}
\operatorname{play}(X): \operatorname{lic}(X, \Delta, B) \rightarrow \operatorname{lic}\left(X, \Delta, B^{\prime}\right) \\
\Leftarrow \Delta \vdash \operatorname{canplay}\left(B, B^{\prime}\right)
\end{array}
$$

1. Suppose the environment requests the action play(music). This will match rule $\operatorname{play}(X)$, giving matching $\sigma_{1}=\{X /$ music $\}$.

2. The next step involves looking for occurrences of lic(music, $\Delta, B)$ in $M S$. The only possible match is $\operatorname{lic}($ music, $\Gamma, C)$. This gives us matching $\sigma_{2}=$ $\{\Delta / \Gamma, B / C\}$.

3. Condition $\Gamma \vdash \operatorname{canplay}\left(C, B^{\prime}\right)$ has to be evaluated. Since variable played_times is less than 10 in $C$, the canplay $\left(C, B^{\prime}\right)$ succeeds in the Prolog program $\Gamma$, hence the condition is satisfied. We get the computed answer substitution $\delta_{1}=\left\{B^{\prime} /\{\right.$ played_times $\equiv 3\}$.

4. Finally, $M S$ is updated. License $\operatorname{lic}($ music, $\Gamma, C)$ is removed from $M S$, and replaced by lic(music, $\left.\Gamma, C^{\prime}\right)$, where $C^{\prime}=\{$ played_times $\equiv 3\}$.

Example 6. Consider the same multiset and rules of the previous example. Suppose now request action play (video) is issued. This action, even though has a matching rule and a matching license in the multiset, cannot be carried out completely. This is so since, in the unique matched license (that is, lic(video, $\Sigma, D)$ ) condition $\Delta \vdash \operatorname{canplay}\left(B, B^{\prime}\right)$ does not hold.

Definition 2.4 describes how a multiset can evolve to another by means of executing a rule. The precise notion of multiset execution, which can be understood as the semantics of LicenseScript, can be then described as sequences of rule executions.

Definition 7. Given a multiset $M S$ and a set of rules $R$, an execution is the (possible infinite) sequence of rule applications $M S \stackrel{a_{1} \sigma_{1}}{\longrightarrow} M S_{1} \stackrel{a_{2} \sigma_{2}}{\longrightarrow} M S_{2} \ldots$ The trace execution of $M S$ is defined as $a_{1} \sigma_{1} \cdot a_{2} \sigma_{2} \ldots$

Semantics of executions of a multiset and a rule set is then defined as all possible trace executions, according to the above definitions.

\section{Examples in Electronic Music Distribution}

In this section we provide examples in Electronic Music Distribution, showing the flexibility of LicenseScript.

\subsection{Music in and between Authorized Domains}

As explained in the introduction, an authorized domain can be seen as the collection of devices belonging to a household. In this paper we only focus on the relationship between licenses and ADs, and we assume the existence of an $\mathrm{AD}$ implementation that deals with domain and content management issues (see [14]), i.e. we assume the presence of compliant devices that are governed by $\mathrm{AD}$ management rules.

We first show how a license can be bound to a specific domain. Consider the following license:

$$
\text { lic }(\text { mus }, \Delta,\{\text { in_domain } \equiv \text { cert }\})
$$

where

$$
\begin{aligned}
& \Delta=\left\{\text { validdomain }(B):-\quad \text { identify }\left(I d_{1}\right)\right. \text {, } \\
& \text { get_value }\left(B, \text { in_domain, } I d_{2}\right) \text {, } \\
& \left.I d_{1}=I d_{2} \cdot\right\}
\end{aligned}
$$

Here, identify $\left(I d_{1}\right)$ is a primitive which is used to retrieve the identity of the current domain. Clause validdomain $(\cdot)$ checks that the current domain is in fact the domain to which the license is bound. A rule like play $(\cdot)$, for instance, can now be defined as only valid if the license is in the allowed domain:

$$
\begin{aligned}
\operatorname{play}(\text { Mus }) & : \operatorname{lic}(\text { Mus }, \Delta, B) \rightarrow \operatorname{play}(M u s, \Delta, B) \\
\Leftarrow & \Delta \vdash \text { validdomain }(B)
\end{aligned}
$$

Now we are ready to illustrate a slightly more complex example: Consider a license that allows a certain piece of music to be played only for a limited time within the domain. This license can be written as follows:

lic(mus, $\Delta,\{$ in_domain $\equiv$ cert, expires $\equiv$ expiration_date $\})$

where $\Delta$ consists of the following two clauses:

$$
\begin{aligned}
& \operatorname{validdomain}(B) \quad:-\quad \text { identify }\left(I d_{1}\right) \text {, } \\
& \text { get_value }\left(B, \text { in_domain, } I d_{1}\right) \text {, } \\
& I d_{1}=I d_{2} \text {. } \\
& \text { notexpired }(B) \text { : - get_value }(B \text {,expires, Exp), } \\
& \operatorname{today}(D), D \leq E x p \text {. }
\end{aligned}
$$

Finally, the corresponding play(·) rule can be defined:

$$
\begin{aligned}
\operatorname{play}(\text { Mus }) & : \operatorname{lic}(\text { Mus }, \Delta, B) \rightarrow \operatorname{play}(M u s, \Delta, B) \\
& \Leftarrow \Delta \vdash \operatorname{validdomain}(B), \Delta \vdash \operatorname{notexpired}(B)
\end{aligned}
$$

\subsection{Payment}

To address the different forms of payment, we first model a wallet. Then, we show how various payment methods are implemented in LicenseScript. 


\subsubsection{Modelling a Wallet}

We can model the existence of a wallet, in our approach, as a another element of the multiset. The wallet is represented as a term of the form wallet $(\Delta, B)$, where $\Delta$ is a Prolog program, and $B$ are a set of bindings. Similarly to licenses, in the wallet we have clauses that allow rules to perform operations over the wallet. We assume that one binding named $m$, which represents the amount of money in the wallet, is always in $B$.

A clause that may reside in $\Delta$ is $\operatorname{canload}(\cdot)$, which is used to load or increase the balance of the wallet, as can be shown as follows:

$$
\begin{aligned}
\operatorname{canload}\left(A, B, B^{\prime}\right):- & \operatorname{get} \_v a l u e(B, m, M), \\
& \text { set_value }\left(B, m, M+A, B^{\prime}\right) .
\end{aligned}
$$

where $A$ is the amount of money the user likes to insert to the wallet.

Using clause $\operatorname{canload}(\cdot)$, a rule that loads money into the wallet can now be written:

$$
\begin{aligned}
\operatorname{load}(\text { Amount }) & : \quad \operatorname{wallet}(\Delta, B) \rightarrow \operatorname{wallet}\left(\Delta, B^{\prime}\right) \\
& \Leftarrow \Delta \vdash \operatorname{canload}\left(\text { Amount }, B, B^{\prime}\right)
\end{aligned}
$$

Another useful clause in the wallet is cantransfer $(\cdot)$, used to transfer a certain amount of money to another entity (e.g., a content provider):

$$
\begin{aligned}
\text { cantransfer }\left(P, A, B, B^{\prime}\right):- & \text { get_value }(B, m, M), \\
& A \leq M, \\
& \text { set_value }\left(B, m, M-A, B^{\prime}\right), \\
& \text { transfer }(P, A) .
\end{aligned}
$$

where primitive $\operatorname{transfer}(P, A)$ models the money transfer to entity $P$ of the amount of money $A$.

\subsection{Payment methods}

There are at least three common alternatives of payment, as described in [6]:

- pay per-use: A payment is issued each time the content is used.

- pay upfront: The content can be used after the payment has taken place, for a period of time $p$.

- pay flatrate: The content is used, and then the payment must be made at the end of the content usage.

We now illustrate the implementation of the pay per-use and pay upfront methods in LicenseScript. (We leave aside pay flatrate, as it is similar to pay per-use.)
Pay Per Use We can model pay per-use in LicenseScript by means of including in a license the following clause canplay $(\cdot)$ :

$$
\begin{aligned}
\operatorname{canplay}(P, A, B):- & \operatorname{get} \text { _value }(B, \text { provider }, P), \\
& \text { get_value }(B, \text { amount }, A) .
\end{aligned}
$$

Here, binding provider is the binding representing the content provider, while binding Amount represents the cost to play music track. Intuitively, clause canplay returns the price of the content in Amount and the provider who should receive the payment, in variable Provider. This allows a rule calling this clause to perform the required payment:

$$
\begin{aligned}
\operatorname{play}(\text { Mus }): & \operatorname{lic}(\text { Mus }, \Delta, B), \text { wallet }(\Gamma, C) \\
& \rightarrow \operatorname{lic}(\text { Mus }, \Delta, B), \text { wallet }\left(\Gamma, C^{\prime}\right) \\
\Leftarrow & \Delta \vdash \text { canplay }(\text { Provider, Amount }, B), \\
& \Gamma \vdash \text { cantransfer }\left(\text { Provider }, \text { Amount }, C, C^{\prime}\right)
\end{aligned}
$$

Here, clause canplay(Provider,Amount,B) retrieves Provider and Amount, which clause cantransfer(Provider, Amount, $C, C^{\prime}$ ) uses to perform the actual money transfer.

Pay Upfront For modelling this payment method, we need to use two different clauses and rules, since the actual payment and content usage may differ in time: the payment is first done, and only later the content is used.

Paying. We first define a clause canpay $(\cdot)$ as follows:

$$
\begin{aligned}
\operatorname{canpay}\left(P, A, B, B^{\prime}\right):- & \text { get_value }(B, \text { paid,Paid }), \\
& \text { Paid }=\text { false, } \\
& \text { get_value }(B, \text { provider }, P), \\
& \text { get_value }(B, \text { amount }, A), \\
& \text { today }(D), \\
& \text { set_value }\left(B, \text { period }, D+f p, B^{\prime}\right), \\
& \text { set_value }\left(B, \text { paid }, \text { true }, B^{\prime}\right) .
\end{aligned}
$$

Here, binding paid is a flag that represents whether the content has already been paid or not. Binding period is used to store the allowed period of time for playing the content, and constant $f p$ represents the period of time in which the content can be accessed after the payment.

Clause canpay(Provider,Amount, $B, B^{\prime}$ ) first checks that the payment has not been done yet. It then returns the value of the provider and the amount in variables Provider and Amount. After this, the period of allowed use is set appropiately, and finally flag paid is set to true, indicating the payment. 
Using the above clause we can define the rule for pay upfront:

$$
\begin{aligned}
\operatorname{pay}(\text { Mus }): & \operatorname{lic}(\text { Mus }, \Delta, B), \text { wallet }(\Gamma, C) \\
& \rightarrow \operatorname{lic}\left(\text { Mus }, \Delta, B^{\prime}\right), \text { wallet }\left(\Gamma, C^{\prime}\right) \\
\Leftarrow & \Delta \vdash \text { canpay }\left(\text { Provider }, \text { Amount }, B, B^{\prime}\right), \\
& \Gamma \vdash \text { cantransfer }\left(\text { Provider }, \text { Amount }, C, C^{\prime}\right)
\end{aligned}
$$

Playing. Now, we can define the canplay (.) clause, which will allow the content to be played only if the payment has been done, and the allowed period of time has not expired:

$$
\begin{aligned}
\operatorname{canplay}(B):- & \text { get_value }(B, \text { paid,Paid }), \\
& \text { Paid }=\text { true }, \\
& \text { today }(D), \\
& \text { get_value }(B, \text { period }, P), \\
& D \leq P .
\end{aligned}
$$

Finally, we can define the rule for pay-upfront play (.):

$$
\begin{aligned}
\operatorname{play}(M u s) & : \operatorname{lic}(M u s, \Delta, B) \rightarrow \operatorname{lic}(M u s, \Delta, B) \\
& \Leftarrow \Delta \vdash \operatorname{canplay}(B)
\end{aligned}
$$

\subsection{Clipping Licenses}

Suppose a user who has purchased a music track from a content provider requires some comments from other users. In LicenseScript, she can clip the license and thus the content, and then she can send the clipped licenses to other people as a preview or recommendation. Notice that this operation splits the license but not the content.

The license in question looks like this:

$$
\text { lic }(\text { mus }, \Delta,\{\text { start } \equiv 0, \text { end } \equiv \text { mus_length }\})
$$

where $\Delta$ contains the following $\operatorname{canclip}(\cdot)$ clause:

$$
\begin{aligned}
\operatorname{canclip}\left(S, E, B, B^{\prime}\right):- & \text { get_value }(B, \text { start }, O S), \\
& \text { get_value }(B, \text { end }, O E), \\
& S<O S, E<O E, \\
& \text { set_value }\left(B, \text { start }, S, B^{\prime}\right), \\
& \text { set_value }\left(B, \text { end, } E, B^{\prime}\right) .
\end{aligned}
$$

Bindings start and end are markers that indicate the head and tail of the music track.

The corresponding rule for clip operation can now be defined:

$$
\begin{aligned}
\operatorname{clip}(S, E, M u s): & \operatorname{lic}(M u s, \Delta, B) \rightarrow \\
& \operatorname{lic}(M u s, \Delta, B), \operatorname{lic}\left(M u s, \Delta, B^{\prime}\right) \\
\Leftarrow & \Delta \vdash \operatorname{canclip}\left(S, E, B, B^{\prime}\right)
\end{aligned}
$$

Note that the content is still the same, full-length here; only the start and end markers are modified. A different clip operation that includes the actual production of a new, clipped content, would need the use a primitive that performs the operation.

Duplicating licenses A special case of the clipping operation occurs when we want to duplicate a license. This operation is often needed. In fact, one of the primary requirements of licensescript architecture is that devices don't have to be always on: in particular, we do not want the system to be dependent from the reacheability and the availability of domain server. To implement correctly the concept of authorized domain, we then have to be able to duplicate licenses. Consider for instance the situation of a person that has the license for listening to a piece of music within his home and who rightfully wants to listen to it also while driving her car. If devices are not always on, then the car device might be uncapable of checking on the home server for the presence of the right license. Therefore, there has to be a license for the music in the car device as well, and this is possible only if we can duplicate licenses.

\section{Conclusions and Future Work}

We propose LicenseScript, a novel digital rights language based on multiset rewriting and logic programming. We present the design of the language using a scenario that represents an elaborate pattern of use of content.

LicenseScript differs from other DRLs in that it has an explicit static and dynamic part. The terms and conditions on content form the static part. These terms and conditions usually derive from legal, regulatory and business rules, and are therefore appropriately expressed using Pro$\log$ clauses [13]. A license is used in a changing context and must therefore have the ability to evolve. The dynamics are represented by interpreting a license as an element of a multiset to which multiset rewrite rules are applied. These rules represent the way in which the context (devices and systems) act upon licences. The dual nature of a license (static versus dynamic) is thus represented by a two-tier structure of LicenseScript. The two levels are linked by a set of bindings that represents the current state of the evolution.

As future work, we plan to implement the language, using an existing DRM platform [4]. Furthermore, we plan to study in detail relevant legal, regulatory and business cases to ensure that the language is convenient to use. Licenses evolution is an interesting issue, and we plan to investigate this point further. Formal verification of license properties (e.g., safety) in a given multiset and rule set, similar to the work of [5], could allow us to reason more precisely about what a license is supposed to achieve and what actually allows. 


\subsection{Related Work}

In this section, we briefly discuss the related work.

Gunter et al. [6] from InterTrust Technologies Corporation and Pucella and Weissman [12] from Cornell University have presented two logics for licenses. Gunter et al. by borrowing techniques from programming semantics [7], have developed a model and a language for describing licenses. Their logic consists of a domain of sequences of events called realities. In their logic, an event is modelled as a pair of a time value and an action. Two kinds of action have been envisaged: render $[w, d]$ and pay $[x]$. Here $w$ denotes the copyrighted work (content); $d$ represents a device; and $x$ is a decimal number, representing the amount of payment. render $[w, d]$ denotes the action of rendering the work $w$ on device $d$. pay [x] symbolizes the action of paying an amount of $x$ for using the work. Note that only one event is allowed at a time. A finite set of events is embodied in a reality. A license, then, is a set of realities. Most licenses consist of infinitely many realities in order to allow the use of a work at one or more of infinitely many times during some period.

Using the proposed model, Gunter et al. have formularized several standard license types, which they call simple licenses. They are the same that we have treated in this paper: simple licenses are "Up Front", "Flat Rate" and "Per Use". Simple licenses can be used as the building blocks of more complex licenses.

Pucella and Weissman follow up Gunter et al.'s effort [12]. It can be summarized by the following items:

- There are 4 standard domains: (1) $\mathcal{N}$ for license names (every license is assigned a name), (2) $\mathcal{W}$ for works (copyrighted works), (3) $D$ for devices (for rendering works), (4) $\mathcal{A}$ for atomic actions (which is a union of the render and the pay action).

- There are 3 syntactic categories: (1) action expression, (2) license, (3) formula.

- An action expression $\alpha$ is composed of action-name pairs (i.e. pairs of $(a, n)$ where $a \in \mathcal{A}$ and $n \in \mathcal{N}$ ). Action expressions are either permitted $(P \alpha)$ or obligatory $(O \alpha)$. (This distinction is what makes their logic more accessible and complete than Gunter et al.'s.)

- A license $l$ is an action sequence (not to be confused with an action expression).

- A formula is made up of $n: l$ terms and $\alpha$ terms. $n$ : $l$ means the action sequence $l$ is valid for the license labelled $n$.

- A run $r$ associates a time $t$ with the licenses issued at that time and the actions performed by the client at that time. At most, one action per time per license can occur.

- An interpretation $\pi$ is a tuple $(P, O)$ where $P$ is a permission assignment and $O$ is an obligation assignment. Simply speaking, if $(a, n) \in P(t)$, then action $a$ is permitted by license $n$ at time $t$. Similar intuition applies to $O(t)$.

- The consistency notion says that if an interpretation $\pi$ enforces all the permissions and obligations required by the licenses issued by a run $r$, then $\pi$ is consistent with $r$. In other words, checking for license violation in a run boils down to checking whether the prevailing interpretation is consistent with the run.

Pucella et al.[12] consider the scenario of an owner of an online journal requiring a fee to be paid before each access. This scenario is similar to the pay-per-use operation that we have shown in section 3.2, with a slight dissimilarity: the users must pay before even activates the application to read the digital journal (our payment is activated when the user activates the application).

Pucella et al.'s license is written as follows:

$$
l=\left(\left(\text { pay }[\text { fee }](\perp)^{*} \text { render }[\text { journal }, d]\right) \cup \perp\right)^{*}
$$

where $d$ is the device that the user uses to access the journal; $\perp$ represents the null or "do nothing" action; pay[fee] is the action of paying amount fee; render $[j o u r n a l, d]$ is the action of accessing the journal using the device $d$ (Refer to Reference [12] for more details on their logic).

Notice that the notations we apply in the following examples refer to section 3.2. We can express the similar license by using the LicenseScript language:

$$
\begin{array}{r}
\text { lic }(\text { journal }, \Delta,\{\text { wallet } \equiv m, \text { paid } \equiv \text { false }, \\
\text { rate } \equiv n, \text { provider } \equiv \text { cert }\})
\end{array}
$$

To express the pay and render operation, we may adopt our pay per-use as discussed in section 3.2. We build the license clauses, $\Delta$ as follows:

$$
\begin{aligned}
\text { canrender }(P, A, B):- & \text { get_value }(B, \text { provider }, P), \\
& \text { get_value }(B, \text { amount }, A) .
\end{aligned}
$$

Hence, we construct the rule to render the journal, paying each time the journal is rendered, by using the clause cantransfer introduced in section 3.2 as follows:

$$
\begin{aligned}
\text { render }(\text { Journal }): & \text { lic }(\text { Journal }, \Delta, B), \text { wallet }(\Gamma, C) \\
& \rightarrow \text { lic }(\text { Journal }, \Delta, B), \text { wallet }\left(\Gamma, C^{\prime}\right) \\
\Leftarrow & \Delta \vdash \text { canrender }(B, \text { Pro, Rate }), \\
& \Gamma \vdash \text { cantransfer }\left(\text { Rate }, \text { Pro }, C, C^{\prime}\right)
\end{aligned}
$$


Acknowledgements. We would like to thank Ernst-Jan Goedvolk, from Telematica Institute for his help on this paper. This work was partially supported by the Telematica Institute.

\section{References}

[1] K. R. Apt. From Logic Programming to Prolog. International Series in Computer Science. Prentice Hall, 1997.

[2] J-P. Banâtre, P. Fradet, and D. L. Métayer. Gamma and the chemical reaction model: Fifteen years after. In C. Calude, G. Paun, G. Rozenberg, and A. Salomaa, editors, Workshop on Multiset Processing (WMP), volume 2235 of Lecture Notes in Computer Science, pages 17-44. Springer-Verlag, Berlin, August 2001.

[3] M. R. V. Chaudron and E. D. de Jong. Towards a compositional method for coordinating gamma programs. In Coordination Languages and Models, First International Conference (COORDINATION '96), pages 107-123. Lecture Notes in Computer Science 1061, Springer-Verlag, April 1996.

[4] C. N. Chong, R. van Buuren, P. H. Hartel, and G. Kleinhuis. Security attributes based digital rights management. In F. Boavida, E. Monteiro, and J. Orvalho, editors, Joint Int. Workshop on Interactive Distributed Multimedia Systems / Protocols for Multimedia Systems (IDMS/PROMS), volume LNCS 2515, pages 339-352, Coimbra, Portugal, Nov 2002. Springer-Verlag, Berlin.

[5] R. Corin and S. Etalle. An improved constraint-based system for the verification of security protocols. In G. Puebla M. Hermenegildo, editor, 9th International Symposium, SAS 2002, volume 2477 of Lecture Notes in Computer Science, pages 326-342. Springer, 2002.

[6] C. Gunter, S. Weeks, and A. Wright. Models and languages for digital rights. In Proceedings of the 34th Annual Hawaii International Conference on System Sciences (HICSS-34), pages 4034-4038, Maui, Hawaii, United States, January 2001. IEEE Computer Society Press.

[7] C. A. Gunter. Semantics of Programming Languages: Structures and Techniques. MIT Press, 1992. ISBN: 0262071436.

[8] H. Guo. Digital rights management (DRM) using XrML. In T-110.501 Seminar on Network Security 2001, page Poster paper 4, 2001. http://www.tml.hut.fi/Studies/T110.501/2001/papers/.
[9] R. Iannella. Open digital rights management. In World Wide Web Consortium (W3C) DRM Workshop, page Position paper 23, January 2001. http://www.w3.org/2000/12/drm-ws/pp/.

[10] J. W. Lloyd. Foundations of Logic Programming. Symbolic Computation - Artificial Intelligence. Springer-Verlag, Berlin, 1987. Second edition.

[11] D. K. Mulligan, A. Burstein, and J. Erikson. Supporting limits on copyright exclusivity in a rights expression language standard. Comments and requirements, Samuelson Law, Technology \& Public Policy Clinic and Clinic and the Electronic Privacy Information Center, Boalt Hall, School of Law, Berkeley CA 94720-7200, USA, August 2002.

[12] R. Pucella and V. Weissman. A logic for reasoning about digital rights. In IEEE Proceedings of the Computer Security Foundations Workshop, pages 282-294, Cape Breton, Nova Scotia, Canada, June 2002. IEEE Computer Society Press.

[13] M. J. Sergot, F. Sadri, R. A. Kowalski, F. Kriwaczek, P. Hammond, and H. T. Cory. The british nationality act as a logic program. Communications ACM, 29(5):370-386, May 1986.

[14] S.A.F.A. van den Heuvel, W. Jonker, F.L.A.J. Kamperman, and P.J. Lenoir. Secure content management in authorised domains. In The World's Electronic Media Event IBC 2002, Sept. 13-17, Amsterdam RAI, The Netherlands, September 2002. To appear. 